\title{
Prevalência de más oclusões e alterações funcio- nais entre escolares assistidos pelo Programa Saú- de da Família em Juazeiro do Norte, Ceará, Brasil
}

André Alencar Suliano*, Paola Colares de Borba**, Maria José Rodrigues***,

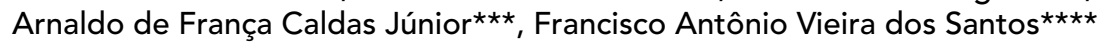

\section{Resumo}

Objetivos: determinar a prevalência de más oclusões e alterações funcionais entre escolares assistidos pelo Programa Saúde da Família em Juazeiro do Norte. Metodologia: realizou-se um estudo transversal na área assistida pela Unidade de Saúde da Família (USF) n ${ }^{\circ} 20$ de Juazeiro do Norte. A partir de uma população de 704 crianças de ambos os gêneros com idades entre 6 e 12 anos, calculou-se a amostra utilizando-se um intervalo de confiança de 95\%. Avaliouse, clinicamente, os padrões oclusal e funcional do sistema estomatognático de 84 crianças, as quais foram convocadas aleatoriamente por agentes comunitárias de saúde. Os padrões foram classificados em: normal (oclusão e funções normais) ou alterado (presença de má oclusão e/ou alteração funcional). A oclusão dividia-se nas relações: vertical, transversal e ântero-posterior. As funções analisadas foram: respiração e deglutição. Para a análise estatística, utilizaram-se os testes Qui-quadrado e de Fisher. Resultados: 77,3\% e 72,6\% da amostra apresentaram más oclusões e padrão funcional alterado, respectivamente; $59,52 \%$ apresentaram ambas as alterações; $45,2 \%$ apresentaram alterações oclusais verticais; $60,7 \%$ alterações transversais e 20,2\% alterações ântero-posteriores; $60,7 \%$ alterações respiratórias e 47,6\% deglutição alterada. Houve associação estatisticamente significativa entre a relação vertical e as duas funções avaliadas $(\mathrm{p}<0,001)$ e entre a relação transversal e a função respiratória $(\mathrm{p}<0,05)$. Conclusões: as prevalências de más oclusões e de alterações funcionais foram elevadas, $77,3 \%$ e $72,6 \%$, respectivamente. Houve uma forte associação entre a relação oclusal vertical e as funções avaliadas (respiração e deglutição).

Palavras-chave: Prevalência. Más oclusões. Alterações funcionais. Programa Saúde da Família.

* Cirurgião-dentista (C.D.), Especialista em Saúde da Família (Faculdade de Medicina de Juazeiro do Norte - FMJ), Mestre e Doutorando em Saúde Coletiva pela Faculdade de Odontologia de Pernambuco.

** Médica, Mestre em Nutrição Humana em Saúde Pública pela London School of Hygiene and Tropical Medicine. Professora do Curso de Especialização em Saúde da Família da FMJ.

*** Professor Doutor do Programa de Pós-graduação da FOP/UPE.

**** C.D., Especialista em Saúde da Família (FMJ), vinculado ao Programa Saúde da Família de Juazeiro do Norte - CE. 


\section{INTRODUÇÃO E REVISÃO DE LITERATURA}

A boca apresenta várias estruturas e estas, em seu conjunto, desempenham funções importantes para o correto funcionamento do organismo humano. Nesse sentido, ao conjunto formado pelas estruturas (componentes morfológicos) da boca e suas funções (componentes funcionais), denomina-se sistema estomatognático ${ }^{6}$. A presença de qualquer desvio morfológico da normalidade caracteriza-se como uma má oclusão. Portanto, a má oclusão tem conotação morfológica, podendo estar acompanhada de alteração funcional.

As más oclusões constituem o grupo de terceira maior prevalência dentre as patologias bucais, sendo inferior, apenas, à cárie e à doença periodonta ${ }^{44}$. Contudo, a medição desta prevalência tem mostrado-se complexa, os seus resultados considerados controvertidos e difíceis de serem comparados, o que aponta para a necessidade de criação de novos estudos e índices que sejam mais amplamente utilizados ${ }^{34}$.

A dificuldade de se comparar resultados envolvendo más oclusões é realçada por Maia, Costa e Maia $^{16}$, os quais categorizaram a oclusão em: normal, má oclusão e má oclusão em potencial. A má oclusão em potencial seria aquela oclusão que não se enquadrava exatamente como oclusão normal, pela perda de dente ou presença de hábito anormal, nem como má oclusão, pois ainda não exibe alterações ${ }^{16}$.

Além disso, boa parte dos levantamentos tem enfatizado apenas as más oclusões ${ }^{11,16,27,30,39}$, o que é corroborado pela Organização Mundial de Saúde (OMS), cujas orientações, em termos epidemiológicos, baseiam-se no Índice de Estética Dental ${ }^{24}$.

Essa visão predominantemente morfológica do problema das más oclusões prejudica a percepção da relevância dos aspectos funcionais relativos ao sistema estomatognático. Tal relevância pode ser ilustrada através da síndrome do respirador bucal, que está relacionada a problemas respiratórios e alérgicos $^{12}$ e a alterações na postura corporal da coluna cervical e cintura escapular de seus portadores $^{36}$. Percebe-se, portanto, que a severidade proporcionada pelas más oclusões e alterações funcionais tende a ser subestimada em termos epidemiológicos quando sua avaliação se detém na perspectiva morfológica e/ou estética do sistema estomatognático.

Outro fator relacionado a essa problemática consiste na diferença existente entre as necessidades de tratamento observadas pelo cirurgião-dentista e a necessidade auto-percebida pelas pessoas examinadas. Utilizando o Índice proposto pela OMS, Peres, Traebert e Marcenes ${ }^{29}$ verificaram que $71,3 \%$ de sua amostra (315 adolescentes entre 14 e 18 anos de idade) possuíam pelo menos um tipo de má oclusão e, destes, 62,3\% estavam satisfeitos com sua aparência. Tal fato corrobora a limitação dos critérios normativos relacionados à estética para aferir a severidade proporcionada pelas más oclusões e alterações funcionais.

Nesse contexto, motivado pela usual escassez de recursos financeiros e pela conotação predominantemente estética conferida às más oclusões, o Sistema Único de Saúde (SUS) não tem desenvolvido ações relacionadas à prevenção e/ou tratamento das más oclusões, priorizando a atuação preventiva e terapêutica contra as manifestações da cárie, por esta ser a doença bucal mais prevalente. Assim, levando-se em consideração que uma parcela significativa da população brasileira tem acesso a serviços de saúde exclusivamente através do SUS, supõe-se que há um grande contingente de pessoas portadoras de más oclusões e alterações funcionais desprovidas de ações preventivas e de tratamento.

Contudo, para que a atenção à saúde e, mais especificamente, à saúde bucal pelo SUS possa realmente se tornar integral, o que inclui a prevenção e o tratamento de más oclusões e de alterações funcionais, faz-se necessária a existência de meios de aferição epidemiológica que se aproximem cada vez mais da realidade clínica. O presente estudo, por sua vez, encontra-se vinculado a esta perspectiva. Assim, objetivou-se determinar a pre- 
valência de más oclusões e de alterações funcionais, bem como analisar se havia associação entre as referidas alterações.

\section{METODOLOGIA}

Realizou-se um estudo epidemiológico transversal na Unidade de Saúde da Família (USF) n ${ }^{\circ} 20$ de Juazeiro do Norte/CE, envolvendo crianças de ambos os gêneros na faixa etária entre 6 e 12 anos, residentes na área adscrita a esta Unidade. Para a coleta de dados, confeccionou-se um formulário específico de inquérito epidemiológico, que foi testado e aperfeiçoado através de um estudo-piloto com uma amostra reduzida $(n=20)$; os resultados desse piloto foram desprezados da análise final. As avaliações clínicas foram realizadas pelo autor principal desta pesquisa, sendo os seus resultados anotados pelo cirurgião-dentista e Especialista em Saúde da Família responsável pela Atenção à Saúde Bucal da referida USF, o que tornou mais factível a realização do presente trabalho.

A população foi estimada em 704 crianças $(\mathrm{N}=704)$ utilizando-se o cadastro existente no Sistema de Informação da Atenção Básica (SIAB) da Secretaria de Saúde do Município de Juazeiro do Norte. A partir desta quantidade, calculou-se a amostra necessária, estimando-se uma prevalência variável entre $60,0 \%$ e 70,0\%, utilizando-se um nível de confiança de $95 \%$ através do software Epilnfo 2002. Esta amostra seria de 72 crianças, sendo aplicado um fator de correção de 1,2 para superestimar a amostra em 20\% em função de possíveis perdas. No total foram examinadas 84 crianças.

As crianças foram convocadas, aleatoriamente, pelas agentes comunitárias de saúde da referida USF. Os exames clínicos foram realizados individualmente no espaço físico do consultório odontológico da Unidade, sob luz artificial, com a criança sentada em uma cadeira em frente ao examinador e ao anotador, ambos também sentados. Solicitou-se o consentimento dos responsáveis pelas crianças e a sua presença foi permitida em todos os momentos da avaliação clínica. Ao final dos exames, os seus resultados, bem como algumas medidas interceptativas de hábitos bucais deletérios, foram transmitidos aos responsáveis.

A avaliação clínica era iniciada pela avaliação do padrão funcional, que era dividido em: respiração e deglutição. Para que este padrão fosse considerado alterado, fazia-se necessário que, no mínimo, uma destas funções estivesse alterada.

$\mathrm{Na}$ avaliação da função respiratória, foram observados três critérios $^{12}$. O primeiro critério dizia respeito ao selamento labial sem contração muscular voluntária $8,915,20,33,38,40$, sendo a sua presença um dos indicativos de normalidade. $\mathrm{O}$ segundo critério relacionava-se ao tempo em que a criança conseguia respirar com os lábios selados ${ }^{2,22,28}$, sendo considerado normal quando a criança conseguisse respirar deste modo por, no mínimo, um minuto. O terceiro critério consistia na colocação de um pequeno espelho abaixo das narinas da criança ${ }^{18,40}$, solicitando-se que a mesma respirasse normalmente; havendo embaçamento do espelho, este critério era considerado um indicativo de normalidade. Quando no mínimo um desses critérios estava alterado, caracterizava-se a presença de respiração bucal. Em virtude da impossibilidade de realização dos exames complementares otorrinolaringológicos para quantificar a permeabilidade do trato respiratório e definir o tipo específico de alteração respiratória, se existente, ao longo do trato respiratório, optou-se pela utilização dos termos respiração nasal e bucal como indicativos de normalidade e de alteração, respectivamente, uma vez que essa terminologia é utilizada por vários autores $2,8,9,10,12,13,14,15,18,20,21,22,23$, $26,28,32,33,38,40,41,43$. Dentro desse contexto, o estabelecimento da etiologia específica das alterações respiratórias detectadas não foi o objetivo do presente trabalho. Além disso, um quadro clínico de respiração bucal provocado por alterações morfológicas (desvio de septo nasal, por exemplo ou pelo hábito de permanecer com a boca aberta) será o mesmo. Fato este corroborado por Krakauer e Guilherme ${ }^{15}$, ambos fonoaudiólogos, que utilizaram como critério de diagnóstico diferencial entre respiração nasal 
e bucal um somatório mínimo de sinais clínicos específicos da respiração bucal.

Para avaliar a deglutição, solicitou-se que a criança colocasse uma quantidade pequena de água mineral em sua boca e não a engolisse imediatamente; após esta etapa, utilizando-se duas espátulas descartáveis de madeira para desocluir os lábios, solicitava-se à criança que a mesma engolisse a água normalmente. O padrão de deglutição era considerado atípico quando se visualizava a presença de interposição lingual entre as arcadas ou quando a língua pressionava de maneira atípica algum dente anterior.

Em seguida, a oclusão dentária foi analisada com o auxílio das referidas espátulas descartáveis, sendo considerada normal quando todas as suas relações constituintes (vertical, transversal e ântero-posterior) estavam normais. Nesse sentido, para a análise vertical foram consideradas as presenças de: normalidade, mordida em topo, mordida aberta e mordida profunda ${ }^{37}$. Para a análise transversal utilizou os seguintes critérios: normalidade; presença de desvio da linha média; presença de apinhamento (superior e/ou inferior); presença de mordida cruzada (posterior uni ou bilateral) ${ }^{42}$.

Para a análise da relação ântero-posterior, utilizou-se a Classificação de Angle ${ }^{5,31}$, levando-se em consideração a chave molar. Desse modo, foram consideradas alterações ântero-posteriores: Classe I com mordida cruzada anterior; Classe I com protrusão dentária; Classe II, $1^{\text {a }}$ Divisão; Classe II, $2^{\mathrm{a}}$ Divisão; Classe III.

Posteriormente às avaliações clínicas, criou-se um banco de dados, através do software Epilnfo 2000. Para a análise estatística inferencial, adotouse um intervalo de confiança de 95\% (95\% IC) para o cálculo das prevalências de más oclusões e de alterações funcionais. Para a análise da associação existente entre as referidas alterações, foram utilizados o teste exato de Fisher (F) e o teste Quiquadrado $\left(\mathrm{X}^{2}\right)$, adotando-se um ponto de significância estatística de $5 \%$.

\section{DISCUSSÃO}

As definições existentes acerca de oclusão normal referem-se às relações morfológicas existentes entre dentes, seja no arco superior ou inferior ou entre ambos ${ }^{1,5}$. De modo análogo, a caracterização de uma oclusão normal envolve de maneira mais abrangente o sistema estomatognático ${ }^{6,37}$, que, por sua vez, não é composto apenas por elementos dentários ${ }^{21}$. Dada a complexidade deste sistema, é necessária a análise conjunta das bases ósseas e dos tecidos moles, para que, a partir desta análise, se possa fazer com segurança as inferências funcionais com suas devidas comprovações e possíveis inter-relações. Nesse sentido, de encontro à utilização do termo oclusão normal para descrever o estado de normalidade do sistema estomatognático, Bueno ${ }^{6}$ ressalta o significado etimológico do termo oclusão.

O que caracteriza o sistema estomatognático é a dinâmica do abrir-fechar. Analisar uma problemática tão complexa, inspecionando, como é comum, apenas a inter-relação dos planos inclinados

Tabela 1 - Distribuição da amostra em relação ao gênero e à idade em anos.

\begin{tabular}{lcccccccc}
\hline \multicolumn{1}{c}{ Idade } & $\mathbf{6}$ & $\mathbf{7}$ & $\mathbf{8}$ & $\mathbf{9}$ & $\mathbf{1 0}$ & $\mathbf{1 1}$ & $\mathbf{1 2}$ & Total \\
\hline Gênero & & & & & & & & \\
Masculino & 5 & 10 & 6 & 8 & 4 & 4 & 3 & 40 \\
Feminino & 8 & 9 & 7 & 7 & 4 & 2 & 7 & 44 \\
Total & 13 & 19 & 13 & 15 & 8 & 6 & 10 & 84 \\
\hline
\end{tabular}

Tabela 2 - Freqüência e percentuais relativos à normalidade/ anormalidade dos componentes da oclusão dentária e do padrão funcional.

\begin{tabular}{ccc}
\hline Componentes clínicos & Normal & Alterado(a) \\
\hline Oclusão & $19(22,6 \%)$ & $65(77,3 \%)^{*}$ \\
Relação vertical & $46(54,7 \%)$ & $38(45,2 \%)$ \\
Relação transversal & $33(39,2 \%)$ & $51(60,7 \%)$ \\
Relação ântero-posterior & $67(79,7 \%)$ & $17(20,2 \%)$ \\
Padrão funcional & $23(27,3 \%)$ & $61(72,6 \%)^{* *}$ \\
Respiração & $33(39,2 \%)$ & $51(60,7 \%)$ \\
Deglutição & $44(52,3 \%)$ & $40(47,6 \%)$ \\
\hline
\end{tabular}

(*95\% IC: $67,0 \%$ a $85,8 \%$; ${ }^{*} 95 \%$ IC: $63,1 \%$ a $82,8 \%$ ). 
oclusais, significa limitar-se a uma faceta, que é sem dúvida importante, mas que não é única, exclusiva e indispensável. Significa, sobretudo, perder-se num enfoque estático de uma fenomenologia essencialmente dinâmica ${ }^{6}$.

Contudo, segundo Proffit et al. ${ }^{31}$, a classificação de más oclusões de Angle é considerada ideal, pois sendo comprovada ao longo de 100 anos de experiência, provou ser correta, exceto quando existem aberrações no tamanho dos dentes, simplificando de maneira brilhante a oclusão normal.

Em relação aos critérios de aferição das más oclusões, Pereira et al. ${ }^{27}$ afirmam que a mensuração da oclusão dentária em populações humanas tem sido um permanente desafio aos profissionais

Tabela 3 - Associação entre oclusão dentária e padrão funcional.

\begin{tabular}{ccc}
\hline \multirow{2}{*}{ Padrão Funcional } & \multicolumn{2}{c}{ Oclusão } \\
\cline { 2 - 3 } Normal & Normal & Alterada \\
Alterado & $8(9,52 \%)$ & $15(17,85 \%)$ \\
\hline
\end{tabular}

$(F=0,18 ; P=0,12)$.

Tabela 4 - Associação entre as funções analisadas e a relação oclusal vertical.

\begin{tabular}{|c|c|c|c|}
\hline \multirow{2}{*}{ Funções } & \multicolumn{2}{|c|}{ Relação Vertical } & \multirow[t]{2}{*}{ Valor de $\mathbf{P}$} \\
\hline & Normal & Alterada & \\
\hline \multicolumn{4}{|c|}{ Respiratória } \\
\hline Normal & $8(9,52 \%)$ & $15(17,85 \%)$ & \\
\hline Alterada & $11(13,09 \%)$ & $50(59,52 \%)$ & $P=0,001\left(X^{2}=9,67\right)$ \\
\hline \multicolumn{4}{|l|}{ Deglutição } \\
\hline Normal & $33(39,28 \%)$ & $11(13,09 \%)$ & \\
\hline Alterada & $13(15,47 \%)$ & $27(32,14 \%)$ & $P<0,0001\left(X^{2}=15,27\right)$ \\
\hline
\end{tabular}

Tabela 5 - Associação entre a função respiratória e a relação oclusal transversal.

\begin{tabular}{cccc}
\hline Função & \multicolumn{2}{c}{ Relação transversal } & Valor de P \\
\cline { 2 - 4 } Respiratória & Normal & Alterada & \\
Normal & $18(21,42 \%)$ & $15(17,85 \%)$ & \\
Alterada & $15(17,85 \%)$ & $36(42,85 \%)$ & $P=0,012\left(X^{2}=5,30\right)$ \\
\hline
\end{tabular}

de Odontologia e de saúde pública.

Essa problemática é corroborada por Maia, Costa e $\mathrm{Maia}^{16}$, que afirmam que muitos dados sobre más oclusões e oclusão normal, disponíveis na literatura, geralmente não correspondem à realidade clínica. Segundo estes autores, este fato pode decorrer da diferença de metodologia empregada nos vários estudos, da conceituação de má oclusão e da interpretação dos dados coletados. Além disso, Peres, Traebert e Marcenes ${ }^{29}$ verificaram que de 214 adolescentes portadores de pelo menos uma má oclusão, 62,3\% estavam satisfeitos com a aparência. Percebe-se, portanto, que há, também, uma diferença acentuada entre a percepção do cirurgião-dentista e a auto-percepção das pessoas examinadas em termos estéticos.

Tendo em vista tal perspectiva, esta pesquisa teve como objetivo estudar as alterações do sistema estomatognático em seu contexto mais amplo, avaliando, além da oclusão dentária, duas de suas principais funções.

Quanto aos dados oclusais, Furtado, Traebert e Marcenes ${ }^{11}$, utilizando critérios propostos pela OMS para a realização de levantamentos em saúde bucal, verificaram que $79,5 \%$ de sua amostra (264 escolares na faixa etária de 6 a 12 anos) apresentavam oclusão normal, 8,7\% apresentavam más oclusões leves e 11,7\% más oclusões variáveis entre moderadas e severas, perfazendo uma prevalência de más oclusões de $20,4 \%$. Os resultados encontrados por estes autores ${ }^{11}$ diferem drasticamente da prevalência observada na presente pesquisa (77,3\%). Entretanto, Peres, Traebert e Marcenes ${ }^{29}$, utilizando, também, critérios da OMS, verificaram que $71,3 \%$ de sua amostra possuíam, pelo menos, uma má oclusão. Resultado semelhante foi observado por Pires, Rocha e Cangussu ${ }^{36}$, que encontraram uma prevalência de más oclusões de $71 \%$. Ambos os resultados se aproximam do encontrado na presente pesquisa $(77,3 \%)$. Já, Silva Filho, Freitas e Cavassan ${ }^{39}$ encontraram um percentual mais elevado $(88,53 \%)$, enquanto Tomita, Bijella e Franco ${ }^{42}$, acrescentando alguns critérios aos da 
OMS em sua metodologia, encontraram alterações oclusais em $51,3 \%$ no gênero masculino e $56,9 \%$ no gênero feminino. Levando-se em consideração a faixa etária utilizada em seu estudo ( 3 a 5 anos), os resultados desses autores ${ }^{42}$ também diferem dos encontrados na presente pesquisa: $80,0 \%$ no gênero masculino e $75,0 \%$ no gênero feminino.

Por sua vez, Maia, Costa e Maia ${ }^{16}$ avaliaram a oclusão de 1.519 crianças de 3 a 14 anos de idade. Eles dividiram sua amostra em três faixas etárias, correspondentes às dentições decídua ( 3 a 6 anos), mista (7 a 10 anos) e permanente ( 11 a 14 anos). $\mathrm{Na}$ dentição mista, eles encontraram as seguintes ocorrências: $71,53 \%$ de más oclusões e 13,33\% de má oclusão em potencial. Apesar das diferenças quanto às definições de má oclusão e às faixas etárias, os resultados destes autores ${ }^{16}$ são considerados próximos dos encontrados na presente pesquisa $(77,3 \%)$.

Já Capote, Zuanon e Pansani ${ }^{7}$, verificando a severidade de má oclusão entre 930 crianças de 6 a 12 anos, constataram que $85,92 \%$ das crianças apresentaram algum tipo de má oclusão, resultados esses próximos aos observados nesta pesquisa: $77,3 \%$. Tal proximidade nos resultados pode ser atribuída aos critérios metodológicos semelhantes e à faixa etária coincidente com a da presente pesquisa.

Jabur et al. ${ }^{12}$ observaram que $46,5 \%$ dos pesquisados eram respiradores bucais. Nesse contexto, acredita-se que a maior prevalência observada nesta pesquisa $(60,7 \%)$ pode ser justificada, dentre outros fatores, por ter-se utilizado neste estudo uma amostra relativamente pequena (84 crianças), pelos critérios de diagnóstico da respiração bucal terem sido essencialmente morfológicos e por não existir um consenso acerca de critérios de diagnóstico de respiração bucal na literatura, o que implica na ausência de comprovação metodológica específica. Nesse sentido, concorda-se com Paiva et al. ${ }^{25}$, os quais apontam a importância do diagnóstico precoce da síndrome do respirador bucal pelo cirurgião dentista, reforçando-se a relevância de se incluir a avaliação de alterações funcionais em posteriores estudos epidemiológicos.

Baldrighi et al. $^{2}$ verificaram associação entre alterações oclusais e deglutição alterada em 47,2\% e entre alterações oclusais e fonação atípica em $41,0 \%$ de sua amostra. Na presente pesquisa, apesar de ter sido observada associação entre más oclusões e alterações funcionais em $59,5 \%$ da amostra, essa associação não foi estatisticamente significativa. Contudo, houve associação significativa $(\mathrm{p}<0,001)$ entre a relação oclusal vertical e as duas funções avaliadas. Esta associação pode ser justificada pela íntima relação existente entre a dinâmica do abrir e fechar a boca e a morfologia do sistema estomatognático apontada por Bueno $^{6}$, corroborando a necessidade de se avaliar de maneira mais ampla tanto as relações oclusais quanto as funções do referido sistema. Além disso, houve associação estatisticamente significante $(\mathrm{p}<0,05)$ entre a relação oclusal transversal e a função respiratória, que pode ser justificada pelo papel desempenhado pela língua na conformação transversal dos arcos dentários. Quando há uma correta função respiratória (respiração nasal), há o vedamento labial e a língua repousa em contato com o palato, conferindo-lhe uma forma adequada. Entretanto, quando há uma disfunção respiratória, não há vedamento labial e a língua repousa no assoalho bucal, deixando de dar a forma adequada ao palato e permitindo que a pressão dos músculos bucinadores possa contribuir para a formação de atresia maxilar e, conseqüentemente, de alterações oclusais transversais.

Apesar do PSF ser uma realidade que tem beneficiado um grande contingente da população brasileira ${ }^{4}$, não foram encontradas na literatura menções acerca de levantamentos epidemiológicos de más oclusões e alterações funcionais vinculados à atenção básica à saúde ou ao PSF. No entanto, Capote, Zuanon e Pansani ${ }^{7}$ concluíram que medidas preventivas devem ser empreendidas em função da alta ocorrência de más oclusões detectada em seu trabalho $(85,92 \%)$. Conclusão esta corrobora- 
da pela presente pesquisa. Dentro desse contexto, levando-se em consideração a alta prevalência de más oclusões e alterações funcionais encontrada no presente estudo e os princípios do $\mathrm{SUS}^{3}$ e do PSF $4,17,35$, bem como o atual panorama de declínio da prevalência da cárie dentária no Brasil ${ }^{19}$, acredita-se que levantamentos epidemiológicos e medidas preventivas relativas às más oclusões e alterações funcionais devem ser planejadas e colocadas em prática no âmbito da Saúde Coletiva. Nesse sentido, acredita-se que se faz necessária a implantação de ações ortodônticas preventivas e interceptativas para as crianças assistidas pela USF $\mathrm{n}^{\circ} 20$ de Juazeiro do Norte.

\section{CONCLUSÕES}

As prevalências de más oclusões e de alterações funcionais foram elevadas, $77,3 \%$ e $72,6 \%$, respectivamente.

Houve uma forte associação entre a relação oclusal vertical e as funções avaliadas (respiração e deglutição).

\title{
Prevalence of malocclusions and functional alterations among students seem by Health Family Program in Juazeiro do Norte, Ceará, Brazil
}

\begin{abstract}
Objectives: determine the prevalence of malocclusions and functional alterations of the stomatognathic system among students seem by a Health Family Team in Juazeiro do Norte. Methodology: it was a cross sectional studies involving the area of the Health Unit 20 of the Family Health Program in Juazeiro do Norte. The study population was 704 children aged from 6 to 12 years old. The sample considered a power of $95 \%$ confidence interval. The community health workers randomly identified 84 children. Their oclusal and functional clinical patterns were evaluated. The stomatognathic system was classified in normal (occlusion and functions normal) and altered (malocclusion and/or functional alteration). The occlusal pattern was separated in antero-subsequent, transverse and vertical relationships. The functional pattern was divided in breathing and swallowing. Qui-square and Fisher's test were used to assess statistical significance. Results: $77,3 \%$ and $72,6 \%$ of the children presented malocclusions and altered functional pattern, respectively. $59,52 \%$ presented alteration in both patterns. $45,2 \%$ presented vertical alterations, $60,7 \%$ transverse alterations and $20,2 \%$ antero-subsequent alterations. $60,7 \%$ had breathing alterations and $47,6 \%$ had altered swallowing. There was association statistically significant between the vertical relationship and the two functions $(p<0,001)$ and between the transverse relationship and the breathing function $(p<0,05)$. Conclusions: the prevalence of malocclusion and functional alterations were high, $77,3 \%$ and $72,6 \%$, respectively. There was a strong association between the vertical relationship and the evaluated functions (breathing and swallowing)
\end{abstract}

Key words: Prevalence. Malocclusion. Functional alterations. Health Family Program.

\section{REFERÊNCIAS}

1. AAO. Glossary of dentofacial orthopedic terms. American Association of Orthodontics, Orthodontic Glossary. 31 p., 1993 apud BRESOLIN, D. Índices para maloclusões. In: PINTO, V. G. Saúde bucal coletiva. 4. ed. São Paulo: Ed. Santos, 2000. p. 197-302
2. BALDRIGHI, S. E. Z. M. et al. A importância do aleitamento natural na prevenção de alterações miofuncionais e ortodônticas. R Dental Press Ortodon Ortop Facial, Maringá, v. 6, n. 5, p. 111-121, set./out. 2001.

3. BRASIL. Ministério da Saúde. Lei orgânica da saúde. Brasília, DF, 1990.

4. Ministério da Saúde. Guia prático do programa saúde da família. Brasília, DF, 2001. 125 p. Disponível em: <http://dtr2001.saude.gov.br/sps/areastecnicas/bucal/publicacoes/guiapsf/guiapsf.htm>.Acesso em: 15 jul. 2003. 
5. BRESOLIN, D. Índices para maloclusões. In: PINTO, V. G. Saúde bucal coletiva. 4. ed. São Paulo: Ed. Santos, 2000. p. 197-302.

6. BUENO, A. P. F. Introdução às bases cibernéticas da ortopedia dentofacial. 1. ed. Rio de Janeiro: Europa, 1991.

7. CAPOTE, T. S. O.; ZUANON, A. C. C.; PANSANI, C. A. Avaliação da severidade de má oclusão de acordo com o gênero, idade e tipo de escola em crianças de 6 a 12 anos residentes na cidade de Araraquara. R Dental Press Ortodon Ortop Facial, Maringá, v. 8, n. 1, p. 57-61, mar./abr. 2003

8 CHAVES, A. V. O. A. et al. Síndrome de classe II divisão $1^{\text {a }}$ de Angle como conseqüência da respiração bucal. Rev Bras Ciênc Saúde, João Pessoa, v. 3, n. 1/3, p. 87-90, 1999.

9. FALTIN JÚNIOR, K.; FALTIN, R. M. Ortodontia preventiva na saúde bucal. In: KRIGER, L. Promoção de saúde bucal. São Paulo: Artes Médicas, 1999. p. 349-361

10. FARIA, P. T. M. et al. Dentofacial morphology of mouth breathing children. Braz Dent J, Ribeirão Preto, v. 13, n. 2, p. 129-132, 2002.

11. FURTADO, A. TRAEBERT, J. L.; MARCENES, W. S. Prevalência de doenças bucais e necessidade de tratamento em Capão Alto, Santa Catarina. Rev ABO Nac, v. 7, n. 4, p. 226-30, ago./set. 1999

12. JABUR, L. B. et al. Estudo clínico da correlação entre padrão respiratório e alterações ortodônticas e miofuncionais. Rev Odontol UNICID, São Paulo, v. 9, n. 2, p. 105-117, jul./dez. 1997.

13. KAWASHIMA, S. et al. Clinical findings in Jananese children with obstructive sep apnea síndrome: focus on dental findings. J Oral Science, Chicago, v. 41, no. 3, p. 99-103, 1999.

14. KOGA, C. Y. et al. Influência da síndrome do respirador bucal na presença de Estreptococos mutans e imunoglobulinas anti-streptococcus mutans na saliva. Rev Odontol UNESP, São Paulo, v. 25, n. 2, p. 207-216, 1996

15. KRAKAUER, L. H.; GUILHERME, A. Relação entre respiração bucal e alterações posturais em crianças: uma análise descritiva. R Dental Press Ortodon Ortop Facial, Maringá, v. 5, n. 5, p. 85-92, set./out. 2000 .

16. MAIA, F. A.; COSTA, P. A. P.; MAIA, N. G. Má oclusão em potencial. R Dental Press Ortodon Ortop Facial, Maringá, v. 4, n. 1, p. 45-51, jan./fev. 1999.

17. MANFREDINI, M. A. Planejamento em saúde bucal. In: PEREIRA, A. C. et al. Odontologia em saúde coletiva: planejando ações e promovendo saúde. 1. ed. Porto Alegre: Artmed, 2003.

18. MANGANELLO, L. C.; SILVA, A. A. F.; AGUIAR, M. B. Respiração bucal e alterações dentofaciais. Rev APCD, São Paulo, v. 56, n. 6, nov./dez. 2002

19. MARCENES, W.; BÖNECKER, M. J. S. Aspectos epidemiológicos e sociais das doenças bucais. In: BUISCHI, Y. P. Promoção de saúde bucal na clínica odontológica. 1. ed. São Paulo: Artes Médicas, 2000

20. MOCELLIN, L.; CIUFFI, C. V. Alteração oclusal em respiradores bucais. J Bras Ortod Ortop Maxilar, Curitiba, v. 2, n. 7, jan./fev. 1997.

21. MOSES, A. J.; LIEBERMAN, M. The effect of external nasal dilators on blood oxygen levels in dental patients. J Am Dent Assoc, Chicago, v. 134, p. 97-101, Jan. 2003.

22. OLIVEIRA, M. O.; VIEIRA, M. M. Influência da respiração bucal sobre a profundidade do palato. Pró-fono Rev Atualização Científica, Barueri, v. 11, n. 1, p. 13-20, 1999.

23. OEVERLAND, B.; AKRE, $H$.; SKATVEDT, O. Oral breathing in pacients with sleep-related breathing disorders. Acta Otolaryngol, Stockholm, v. 122, p. 651-654, 2002.

24. ORGANIZAĈÃO MUNDIAL DA SAÚDE. Levantamentos básicos em saúde bucal. 4. ed. São Paulo: Ed. Santos, 1999

25. PAIVA, J. B. et al. Identificando o respirador bucal. Rev Assoc Paul Cir Dent, São Paulo, v. 53, n. 4, p. 265-274, jul./ago. 1999.

26. PAROLO, A. M. F.; BIANCHINI, E. M. G. Pacientes portadores de respiração bucal: uma abordagem fonoaudiológica. R Dental Press Ortodon Ortop Facial, Maringá, v. 5, n. 2, p. 76-81, mar./abr. 2000.
27. PEREIRA, A. C. et al. Avaliação de alguns instrumentos para detecção de má-oclusão dentária. Odontol USF, Bragança Paulista, v. 18, n. 1, p. 33-39, jan./jun. 2000.

28. PEREIRA, L. F.; SILVA, A. M. T.; CECHELLA, C. Ocorrência de hábitos orais viciosos e distúrbios fonoarticulatórios em indivíduos portadores de deglutição atípica. Pró-fono Rev Atualização Científica, Barueri, v. 10, n. 1, p. 56-60, 1998

29. PERES, K. G.; TRAEBERT, E. S. A.; MARCENES, W. Diferenças entre autopercepção e critérios normativos na identificação das oclusopatias. Rev Saúde Pública, São Paulo, v. 36, n. 2 p. 230-236, 2002

30. PIRES, D. M.; ROCHA, M. C. S.; CANGUSSU, M. C. T. Prevalência de maloclusãos na dentadura mista em escolares - Salvador/ BA. RBO, Rio de Janeiro, 58. n. 6, p. 414-417, 2001.

31. PROFFIT, W. R. et al. Ortodontia contemporânea. 2. ed. Rio de Janeiro: Guanabara Koogan, 1995

32. QUELUZ, D. P.; GIMENEZ, C. M. M. A síndrome do respirador bucal. Rev CROMG, Belo Horizonte, v. 6, n. 1, jan./abr. 2000.

33. RASKIN, S.; LIMME, M.; POIRRIER, R. La ventilation orale peutelle conduire au syndrome des apnées obstuctives du sommeil? Etude préliminaire. Orthod Fr, Paris, v. 71. p. 27-35. 2000

34. RONCALLI, A. G. et al. Projeto SB2000: uma perspectiva para a consolidação da epidemiologia em saúde bucal coletiva. Rev Bras Odontol Saúde Coletiva, Brasília, DF, v. 1, n. 2 p. 9-25, 2000.

35. RONCALLI, A. G. O desenvolvimento das políticas públicas de saúde no Brasil e a construção do Sistema Único de Saúde. In: PEREIRA, A. C. et al. Odontologia em saúde coletiva: planejando ações e promovendo saúde. 1. ed. Porto Alegre: Artmed, 2003

36. SCHINESTSCK, P. A.; SCHINESTSCK, A. R. A importância do tratamento precoce da má-oclusão dentária para o equilíbrio orgânico e postural. J Bras Ortodon Ortop Facial, Curitiba, v. 3, n. 13 , p. $15-30$, jan./fev. 1998

37. SCHURT, C. Ortopedia funcional dos maxilares: da polaridade à unicidade. 1. ed. São Paulo: Quintessence, 2001.

38. SEGOVIA, M. L. Interaciones entre la odontoestomatologia y la fonoaudiologia. 1. ed. Buenos Aires: Médica Panamericana, 1977

39. SILVA FILHO, O. G.; FREITAS, S. F.; CAVASSAN, A. O. Prevalência de oclusão normal e má oclusão em escolares da cidade de Bauru (São Paulo). Parte I: relação sagital. Rev Odontol USP São Paulo, v. 4, n. 2, p. 130-137, abr./jun. 1990.

40. SONCINI, F.; DORNELLES, S. Respiração: contradições entre as informações dos pais e os resultados da avaliação fonoaudiológica. Rev Fono Atual, São Paulo, n. 11, p. 46-51, 2000.

41. TOMÉ, M. C. MARCHIORI, S. C. Análise eltromiográfica dos músculos orbiculares superior e inferior da boca em crianças respiradoras nasais e bucais durante a emissão de sílabas. Pró-fono Rev Atualização Científica, Barueri, v. 11, n. 1 p. 1-7, 1999

42. TOMITA, N. E.; BIJELLA, V. T.; FRANCO, L. J. Relação entre hábitos bucais e má oclusão em pré-escolares. Rev Saúde Pública, São Paulo, v. 34, n. 3, p. 299-303, jun. 2000

43. UEDA, K. et al. Lip seal estudy of japanese adults with malocclusion. Bull Tokyo Dent Coll, Tokyo, v. 43, no. 2, p. 89-93, May 2002

44. BRESOLIN, D. Índices para maloclusões. In: PINTO, V. G. Saúde bucal coletiva. 4. ed. São Paulo: Ed. Santos, 2000. p. 197-302.

\section{Endereço para correspondência}

André Alencar Suliano

Rua Benfica, 341, Bloco B, Apto. 18. Madalena

CEP: 50720-001 - Recife/PE

E-mail: andre.suliano@gmail.com 